\title{
Adjuvant High-Flow Normobaric Oxygen After Mechanical Thrombectomy for Anterior Circulation Stroke: a Randomized Clinical Trial
}

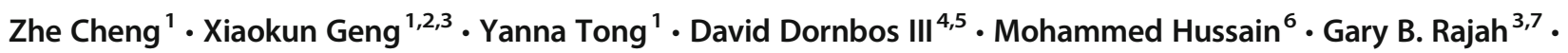 \\ Jie Gao ${ }^{1} \cdot$ Linlin $\mathrm{Ma}^{1} \cdot$ Fenghai $\mathrm{Li}^{1} \cdot$ Huishan $\mathrm{Du}^{1} \cdot$ Marc Fisher $^{8} \cdot$ Yuchuan Ding $^{3,9}$
}

Accepted: 19 November 2020 / Published online: 6 January 2021

(C) The American Society for Experimental NeuroTherapeutics, Inc. 2021

\begin{abstract}
Adjuvant neuroprotective therapies for acute ischemic stroke (AIS) have demonstrated benefit in animal studies, albeit without human translation. We investigated the safety and efficacy of high-flow normobaric oxygen (NBO) after endovascular recanalization in anterior circulation stroke. This is a prospective randomized controlled study. Eligible patients were randomized to receive high-flow NBO by a Venturi mask $\left(\mathrm{FiO}_{2} 50 \%\right.$, flow $\left.15 \mathrm{~L} / \mathrm{min}\right)$ or routine low-flow oxygen supplementation by nasal cannula (flow $3 \mathrm{~L} / \mathrm{min}$ ) after vessel recanalization for $6 \mathrm{~h}$. Patient demographics, procedural metrics, complications, functional outcomes, symptomatic intracranial hemorrhage (sICH), and infarct volume were assessed. A total of 91 patients were treated with high-flow NBO. NBO treatment revealed a common odds ratio of 2.2 (95\% CI, 1.26 to 3.87) favoring the distribution of global disability scores on the $\mathrm{mRS}$ at 90 days. The mortality at 90 days was significantly lower in the NBO group than in the control group, with an absolute difference of $13.86 \%$ (rate ratio, $0.35 ; 95 \% \mathrm{CI}, 0.13-0.93$ ). A significant reduction of infarct volume as determined by MRI was noted in the NBO group. The median infarct volume was $9.4 \mathrm{ml}$ versus $20.5 \mathrm{ml}$ in the control group (beta coefficient, -20.24 ; $95 \% \mathrm{CI},-35.93$ to -4.55 ). No significant differences were seen in the rate of $\mathrm{sICH}$, pneumonia, urinary infection, and seizures between the 2 groups. This study suggests that high-flow NBO therapy after endovascular recanalization is safe and effective in improving functional outcomes, decreasing mortality, and reducing infarct volumes in anterior circulation stroke patients within $6 \mathrm{~h}$ from stroke onset.
\end{abstract}

Key Words Oxygen supplement $\cdot$ neuroprotection $\cdot$ endovascular recanalization $\cdot$ large vessel occlusion $\cdot$ functional prognosis.

Xiaokun Geng

xgeng@ccmu.edu.cn

Yuchuan Ding

yding@med.wayne.edu

1 Department of Neurology and Stroke Intervention and Translational Center (SITC), Beijing Luhe Hospital, Capital Medical University, No. 82 Xinhua South Road, Tongzhou District, Beijing 101149, China

2 China-America Institute of Neuroscience, Beijing Luhe Hospital, Capital Medical University, Beijing, China

3 Department of Neurosurgery, Wayne State University School of Medicine, 550 E Canfield, Detroit, Michigan 48201, USA
4 Department of Neurological Surgery, The Ohio State University Wexner Medical Center, Columbus, Ohio, USA

5 Department of Neurosurgery, University of Tennessee Health Science Center and Semmes-Murphey Clinic, Memphis, Tennessee, USA

6 Department of Neurointerventional Surgery, Wesley Medical Center, Wichita, Kansas, USA

7 Department of Neurosurgery, Munson Healthcare, Traverse City, Michigan, USA

8 Department of Neurology, Beth Israel Deaconess Hospital, Harvard Medical School, Boston, Massachusetts, USA

9 John D. Dingell VA Medical Center, 4646 John R Street (11R), Detroit, Michigan 48201, USA 


\section{Introduction}

Recent advances in endovascular therapy with newgeneration stent retrievers, large bore aspiration catheters, and expanded intervention time windows have achieved high rates of revascularization for acute ischemic stroke (AIS) patients with large vessel occlusion (LVO). Endovascular therapy has demonstrated improvement in functional outcomes in AIS patients with LVO in the anterior circulation $[1,2]$. However, only $46 \%$ of patients treated with endovascular therapy for anterior circulation LVO achieve functional independence at 90 days with a $15.3 \%$ mortality rate $[1,3]$. A relative disconnect exists between recanalization and good functional outcomes with substantial room for improvement. Therefore, additional adjunctive neuroprotective treatment strategies after endovascular recanalization continue to be warranted to mitigate mortality rates and increase good functional outcomes in the acute clinical setting.

Adjuvant neuroprotective therapies following AIS have demonstrated benefit in numerous preclinical animal studies $[4,5]$. However, there has been an overall lack of successful translation of this application to human studies [6]. Many neuroprotectants have only demonstrated benefit in animal stroke models when combined with vessel recanalization, a fact that may underscore the discrepancy between many preclinical models and clinical efficacy $[7,8]$. The recent advances in endovascular therapy for AIS with high rates of complete recanalization have opened a potential opportunity to successfully apply, augment, and translate auxiliary neuroprotective strategies to patients after successful reperfusion [7]. A very recent clinical trial (ESCAPE-NA1) have demonstrated that nerinetide, a neuroprotectant perturbing PSD-95 protein-protein interactions, improved functional outcomes and reduced mortality among non-alteplase AIS patients after mechanical thrombectomy [9]. This study further suggests a promising combination of reperfusion and neuroprotection strategies in AIS patients.

As a traditional neuroprotectant, oxygen supplementation has been suggested to reduce secondary injury to penumbral tissue, minimizing damage and promoting neural recovery and plasticity in AIS [10-14]. Normobaric oxygen (NBO) has gained substantial attention for its clinical ease and feasibility and been recognized as a potential adjuvant neuroprotective therapy in AIS [15]. Animal studies have suggested that NBO treatment may be effective to improve outcomes in ischemic stroke before, during, or after ischemia [13, 14, 16-20]. However, previous clinical trials have failed to demonstrate a consistent neuroprotective effect of NBO in ischemic stroke [21]. Both published phase II RCTs of NBO documented feasibility and safety but found no clinical benefit $[22,23]$. The reason for the failure was considered to be that $\mathrm{NBO}$ was not combined with recanalization in these clinical trials [8]. At present, no study has been reported on the safety and efficacy of NBO as an adjuvant neuroprotective strategy combined with endovascular recanalization in AIS. Given the technique of mechanical thrombectomy has improved over the past decade, reperfusion can now be successfully reestablished in most LVO patients and can provide an avenue for neuroprotectants to reach at-risk tissue. This study was designed initially to determine whether the administration of NBO after recanalization with mechanical thrombectomy conferred a clinical benefit.

\section{Methods}

Participants We conducted a prospective randomized controlled trial (ChiCTR-INR-17013685) to assess the safety and efficacy of NBO administration in AIS patients with anterior circulation LVO after mechanical thrombectomy. A total of 233 stroke patients with LVO in the anterior circulation were screened consecutively in the Stroke Intervention and Translational Center (SITC), Beijing Luhe Hospital Capital Medical University, from December 2017 to December 2019. The protocol was approved by the Beijing Luhe Hospital Research Ethics Committee. The participants gave a fully informed written consent or this was provided by a legal representative if they did not have the capacity to consent.

The trial included patients that met the following criteria: 1) age between 18 and 80 years; 2) clinical diagnosis of AIS; 3) large vessel occlusion in the anterior circulation demonstrated with CTA, MRA, or DSA; 4) a baseline National Institutes of Health Stroke Scale (NIHSS) scores 6-25 and an Alberta stroke program early CT (ASPECT) scores 6-10; and 5) less than $6 \mathrm{~h}$ from stroke onset to groin puncture. Exclusion criteria were as follows: 1) rapid spontaneous improvement in neurological status to an NIHSS $<6$ or evidence of spontaneous vessel recanalization; 2) no vessel recanalization (TICI score $=0$ ) after mechanical thrombectomy; 3) symptoms that rapidly improved after vessel recanalization; 4) brain imaging revealing an infarction greater than one third of the MCA territory; 5) prestroke modified Rankin scale (mRS) > ; 6) active and chronic obstructive pulmonary disease, such as chronic obstructive pulmonary disease, obstructive sleep apnea, and acute respiratory distress syndrome; 7) use of an oxygen face mask or ventilator for the purpose of maintaining appropriate oxygen saturation levels; 8) general anesthesia; 9) severe arterial blood pressure $>$ $185 / 110 \mathrm{mmHg} ; 10$ ) blood glucose $<2.7$ or $>22.2 \mathrm{mmol} /$ 1 ; 11) laboratory evidence of coagulopathy (platelet count $<40 \times 10^{9} / 1$, APTT > $50 \mathrm{~s}$, or INR > 3.0); 12) pregnancy; 13) patients being enrolled or having been enrolled in other clinical trial within 3 months prior to this clinical trial; and 14) informed consent was not obtained. 
Randomization During the recruitment period, participants were allocated 1:1 to 2 groups by computer-generated, randomization procedures. To avoid the possible bias from different visual cues between mask and nasal catheter, patient variables and outcomes, before and after oxygen supplementation, were assessed by 2 groups of independent physicians who were either blinded to the treatment and patient evaluation/data collection. Specifically, the first group was responsible for initial patient screening and performing 6-h oxygen administration. Following oxygen supplementation, case observation, date collection, and statistical analysis were carried out by the second group, including physicians, who were blinded to the treatment. Clinicians and data collectors in the second group, participant families, adjudicators, and data analysts were all blinded to the study.

Intervention Patients who were eligible for intravenous thrombolysis received recombinant tissue plasminogen activator (rtPA) at a dose of $0.9 \mathrm{mg} / \mathrm{kg}$ ( $10 \%$ of the dose was given as a bolus within $1 \mathrm{~min}$ followed by a 60-min total infusion) while endovascular intervention was simultaneously being prepared. All endovascular procedures were performed under local anesthesia with/without sedative agents. Interventional strategies were left to the discretion of the treating interventionalist, including the choice of stent retrievers, aspiration, stenting, or other necessary devices. Routine oxygen by nasal cannula was given during thrombectomy. Following the procedure, patients received supplemental high-flow NBO therapy by a Venturi mask $\left(\mathrm{FiO}_{2} 50 \%\right.$, flow $\left.15 \mathrm{~L} / \mathrm{min}\right)$ or routine low-flow oxygen supplementation by nasal cannula (flow $3 \mathrm{~L} / \mathrm{min}$ ) after vessel recanalization as soon as possible, which was continued for $6 \mathrm{~h}$.

Outcomes Measures All participants had standard assessments of demographic characteristics, medical history, stroke severity, and intervention procedure. The primary outcome measures were mRS score at 90 days, assessed by trained personnel who were blinded to the treatment group assignments. The $\mathrm{mRS}$ is a graded interval scale (range, 0 [no symptoms] to 6 [death]) for the assessment of neurologic functional disability [24]. The secondary outcome measures were as follows: functional independence (mRS scores of 0 to 2), NIHSS score at $24 \mathrm{~h}$, infarct volume, mortality (mRS score 6), symptomatic intracranial hemorrhage ( $\mathrm{sICH}$ ), fatal $\mathrm{ICH}$, pneumonia, urinary infection, and seizures. Seizures in the study was defined as a transient occurrence of sign and/or symptoms due to abnormal excessive or synchronous neuronal activity in the brain. The data on prerandomization oxygen supplementation was not analyzed.

Imaging Assessment A brain computed tomographic (CT) scan was obtained at baseline and immediately after endovascular intervention to assess ischemic stroke burden and ICH. ASPECTS scoring was done on the baseline CT. Magnetic resonance imaging (MRI) was routinely performed at $24 \mathrm{~h}$ after vessel recanalization. If MRI could not be completed or there was any sign that neurological deterioration occurred within $24 \mathrm{~h}$, another head CT scan was performed. Infarct volume was measured on a Siemens Syngo.Via workstation with diffusion-weighted MRI (https://www. siemenshealthineers.com/magnetic-resonanceimaging/ advanced-imaging-applications/syngo-via). Lesions were outlined on each individual slice using the image tool in the workstation to calculate area. The infarct volumes were calculated by multiplying the area of each slice by slice thickness. Target vessel recanalization was assessed by the modified Thrombolysis in Cerebral Infarction (mTICI) scale [25]. ASPECTS and TICI scoring were assessed by 2 neuroradiologists blinded to clinical presentation and treatment group.

Statistical Analysis Sample size was estimated from one previous NBO study [23] based on the difference of mRS score at 90 days between NBO-treated and control groups. A total of 180 patients were calculated as necessary to show a favorable outcome of NBO groups at 90 days after vessel recanalization in AIS. All analysis was performed in the intention-to-treat population. The common odds ratio of the mRS score at 90 days was estimated by using ordinal logistic regression model. The rate ratio of mRS $0-2$, mRS 6 , and adverse events were estimated by using the modified Poisson regression approach [26]. Beta coefficient for infarct volume and NIHSS score were estimated by using multiple linear regression. Estimates of effect were adjusted for age, sex, baseline NIHSS score, baseline ASPECTS, location of occlusion, status with respect to intravenous alteplase treatment, the number of passages of retriever stent, time from treatment to recanalization, and TICI. Analysis of functional independence at 90 days in subgroups was done by the Breslow-Day test for homogeneous odds ratios across subgroups. Two-sided $p<0.05$ values were used throughout the paper. All analyses were done with SAS (Version 9.1.3: SAS Institute, Cary, NC).

\section{Results}

Patients A total of 180 patients were enrolled and randomized in the study. Five participants were lost to follow-up and a total of 175 participants were finally analyzed ( 88 in the NBO group and 87 in the control group) (Fig. 1). Baseline demographic and characteristics of the patients are summarized in Table 1. Age, sex ratio, baseline NIHSS, vascular risk factors, ASPECTS score, location of stroke, IV rt-PA, stent utilization, time from onset to recanalization, time from treatment to recanalization, the number of passages of retriever 
Fig. 1 Flow and timeline of participants enrolled

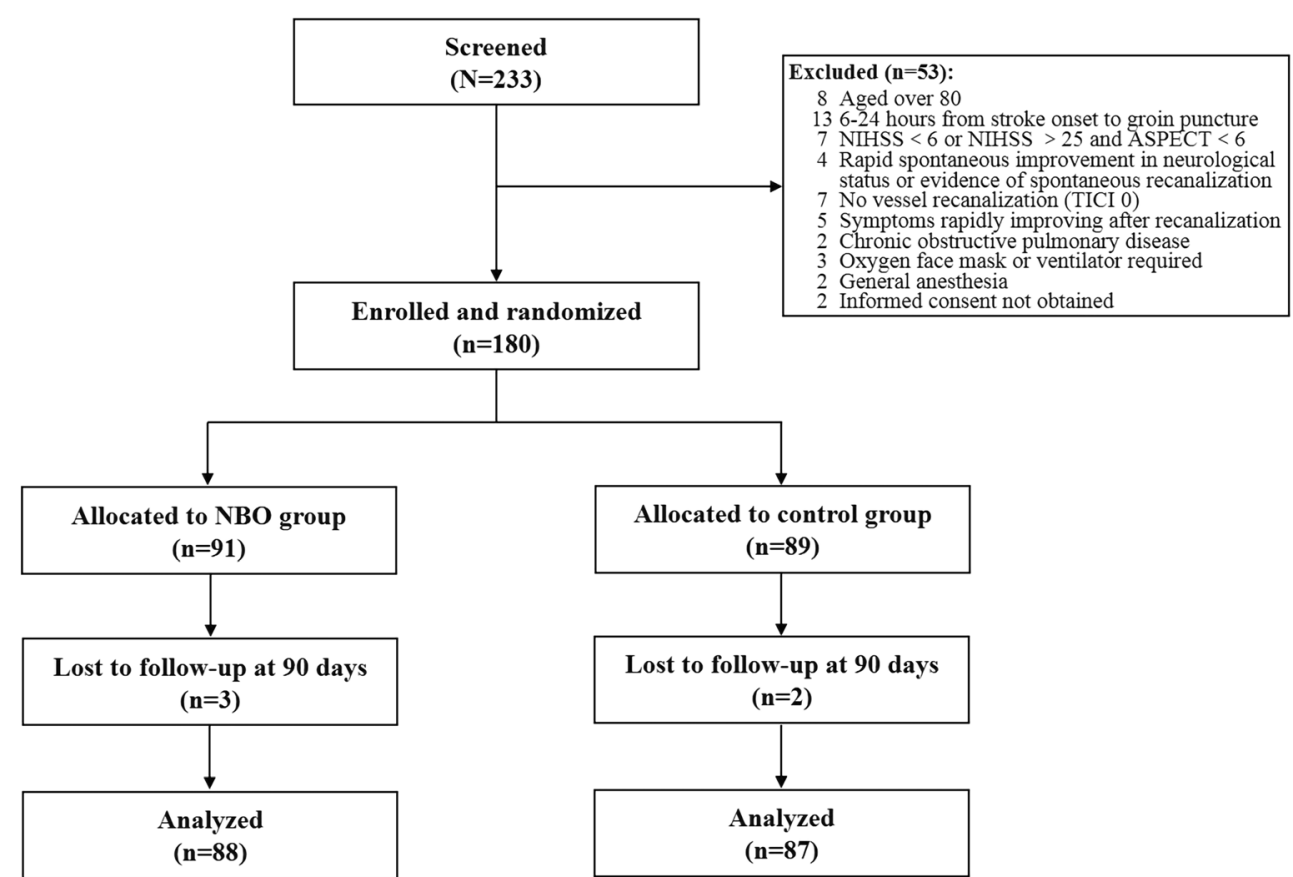

Primary Outcome NBO treatment demonstrated an adjusted common odds ratio (indicating the odds of improvement of 1 stent, and TICI score were not significantly different between the NBO and control groups (Table 1).

\begin{tabular}{lll}
\hline Characteristic & NBO $(n=88)$ & Control $(n=87)$ \\
\hline Age, mean \pm SD, years & $63.8 \pm 11.5$ & $65.9 \pm 10.5$ \\
Sex ratio, no. (\%) & $56(63.6)$ & $51(58.6)$ \\
NIHSS score, median (IQR) & $17(14-18)$ & $16(14-19)$ \\
ASPECTS value, median (IQR) & $9(8-9)$ & $8(8-9)$ \\
Risk factors, no. (\%) & & \\
Hypertension & $57(64.8)$ & $62(71.3)$ \\
Diabetes mellitus & $26(29.5)$ & $20(23.0)$ \\
Atrial fibrillation & $34(38.6)$ & $29(33.3)$ \\
Smoking & $26(29.5)$ & $23(26.4)$ \\
Occlusion site, no. (\%) & & \\
MCA & $50(56.8)$ & $58(66.7)$ \\
Intracranial ICA & $19(21.6)$ & $15(17.2)$ \\
P-ICA & $19(21.6)$ & $41(47.1)$ \\
rt-PA, no. (\%) & $37(42.0)$ & $20(23.0)$ \\
Stent, no. (\%) & $25(28.4)$ & $60(44-78)$ \\
Time from treatment to & $57(40-74)$ & $240(200-305)$ \\
$\quad$ recanalization, median (IQR), min & & \\
Time from onset to recanalization, & $231(181-286)$ & $1(1-2)$ \\
$\quad$ median (IQR), min & & \\
The number of passages of & $1(1-1)$ & $4(4.6)$ \\
$\quad$ retriever stent, median (IQR) & & $10(11.5)$ \\
TICI score, no. (\%) & $3(3.4)$ & $22(25.3)$ \\
1 & $9(10.2)$ & $51(58.6)$ \\
2a & $20(22.7)$ & \\
2b & $56(63.6)$ & \\
3 & & \\
\hline
\end{tabular}

There were no significant differences between the 2 groups

NIHSS = the National Institute of Health Scale Score; ASPECT = Alberta Stroke Program Early Computed Tomography Score; $\mathrm{mRS}=$ modified Rankin score; $\mathrm{MCA}=$ middle cerebral artery segment; ICA = internal carotid artery; P-ICA = proximal segment of internal carotid artery occlusion combined with intracranial large artery occlusion; IV rt-PA = intravenous recombinant tissue plasminogen activator; TICI $=$ thrombolysis in cerebral infarction; IQR = interquartile range 
point on the $\mathrm{mRS}$ ) of 2.2 (95\% CI, 1.26 to 3.87 ) favoring the distribution of global disability scores on the mRS at 90 days $(p=0.006)$ (Fig. 2 and Table 2).

Secondary Outcomes There was an absolute difference between the 2 groups in the proportion of patients who were functionally independent, favoring NBO (14.2\%; adjusted odds ratio, $1.2 ; 95 \% \mathrm{CI}, 0.95$ to 1.52 ), although it did not reach a significant level $(p=0.14)$. However, mortality at 90 days was significantly lower in the NBO group than in the control group, with an absolute difference of 13.86 percentage points (adjusted risk ratio, $0.35 ; 95 \% \mathrm{CI}$, $0.13-0.93 ; p=0.012$ ). Additionally, at $24 \mathrm{~h}$ after stroke onset, a significant reduction of infarct volume determined by MRI was found in the NBO group, and the median infarct volume was $9.4 \mathrm{ml}$ compared with $20.5 \mathrm{ml}$ in the control group (adjusted beta coefficient, $-20.24 ; 95 \% \mathrm{CI}$, -35.93 to $-4.55 ; p=0.035$ ) (Table2 and Fig. 3 ). There was no significant difference between the 2 groups in median NIHSS score at $24 \mathrm{~h}$ after stroke onset (11 vs 14; adjusted beta coefficient, -1.28 ; 95\% CI, $-2.80-0.25$ ). The rate of symptomatic ICH was $6.8 \%$ in the NBO group and $9.2 \%$ in the control group (adjusted rate ratio, 1.06; 95\% CI, 0.44-2.54), with no significant difference between the 2 groups. Similarly, no difference was observed in the rates of fatal ICH $(1.1 \%$ vs $4.6 \%$, adjusted rate ratio, 0.34 ; 95\% CI, $0.02-5.00)$, pneumonia (12.5\% vs $13.8 \%$, adjusted rate ratio, $1.03 ; 95 \% \mathrm{CI}, 0.47-2.27)$, urinary infection $(4.6 \%$ vs $9.2 \%$, rate ratio, 0.49 ; $95 \%$ CI, $0.15-$ $1.58)$, and seizures $(3.4 \%$ vs $3.5 \%$, rate ratio, $0.99 ; 95 \%$ CI, 0.21-4.76) (Table 2).
Subgroup Analysis In the study sample size, no evidence of heterogeneity of treatment effect was detected in any of the prespecified subgroups (defined according to sex, age, baseline NIHSS score, baseline ASPECTS, occlusion location, status with respect to alteplase treatment, and stent). All variables showed a direction of effect in favor of the intervention (Fig. 4).

\section{Discussion}

This study reveals a favorable outcome following administration of high-flow NBO adjuvant therapy after endovascular recanalization in AIS patients with anterior circulation LVO. No increased risk of $\mathrm{ICH}$, pneumonia, urinary infection, and seizures were observed following this therapy. NBO administration significantly improved functional outcome (mRS score) and decreased mortality at 90 days after stroke onset while reducing infarct volume in stroke patients. A recently published paper in Stroke noted reconsidering neuroprotection in the era of reperfusion [8]. Given that mechanical thrombectomy and reperfusion provides neuroprotection to at-risk tissue, it makes endovascular intervention imperative in any trial assessing neuroprotection efficacy in stroke caused by LVO. To our knowledge, this was the first study to report randomized data on safety and efficacy of NBO in AIS patients after successful endovascular recanalization.

Safety of NBO Administration Many studies have shown NBO administration to be safe in AIS patients [21]. However, there are conflicting reports in the literature, wherein some studies have

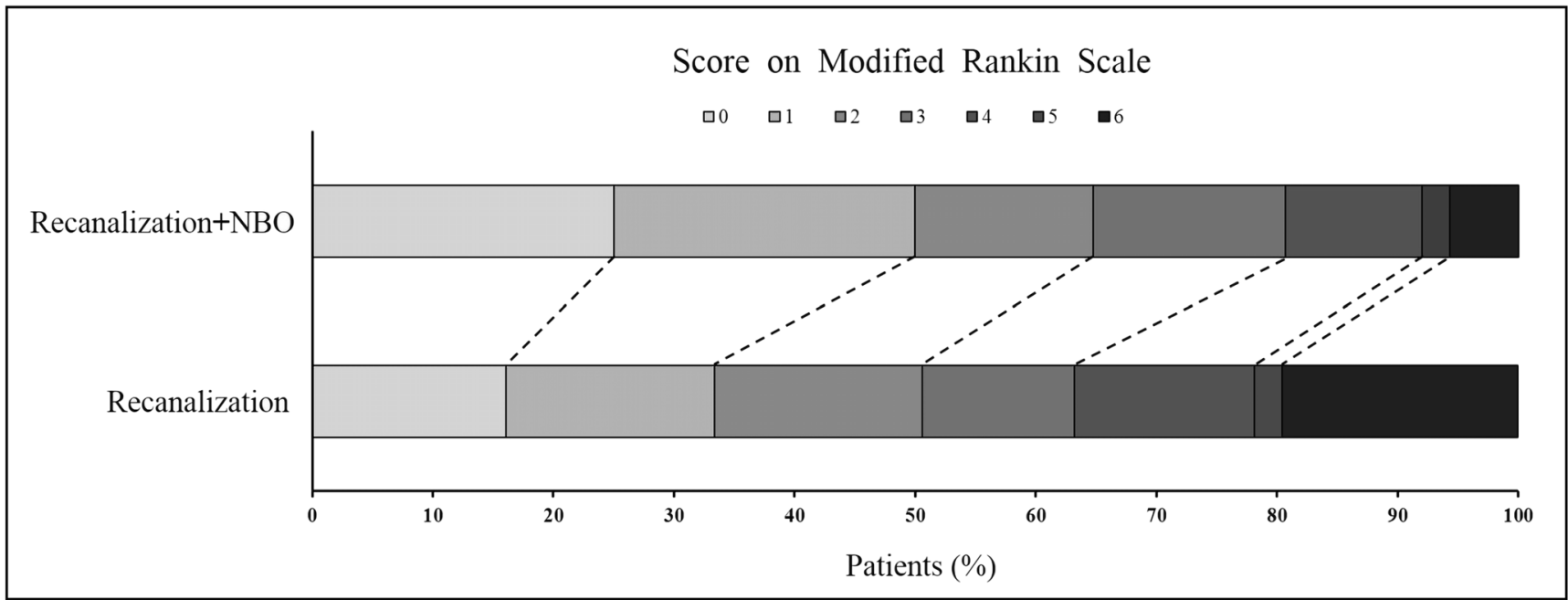

Fig. 2 Modified Rankin Scale score in AIS patients after vessel recanalization at 90 days. Image of scores on the modified Rankin Scale range from 0 to 6 in AIS patients after vessel recanalization at 90 days; 0 indicating no symptoms, 1 no clinically significant disability, 2 slight disability, 3 moderate disability, 4 moderately severe disability, 5 severe disability, and 6 death. Significant difference between the NBO and control groups was noted in the overall distribution of scores (common odds ratio, indicating the odds of improvement of 1 point on the modified Rankin Scale, 2.2; 95\% confidence interval, 1.26 to 3.87), favoring NBO 


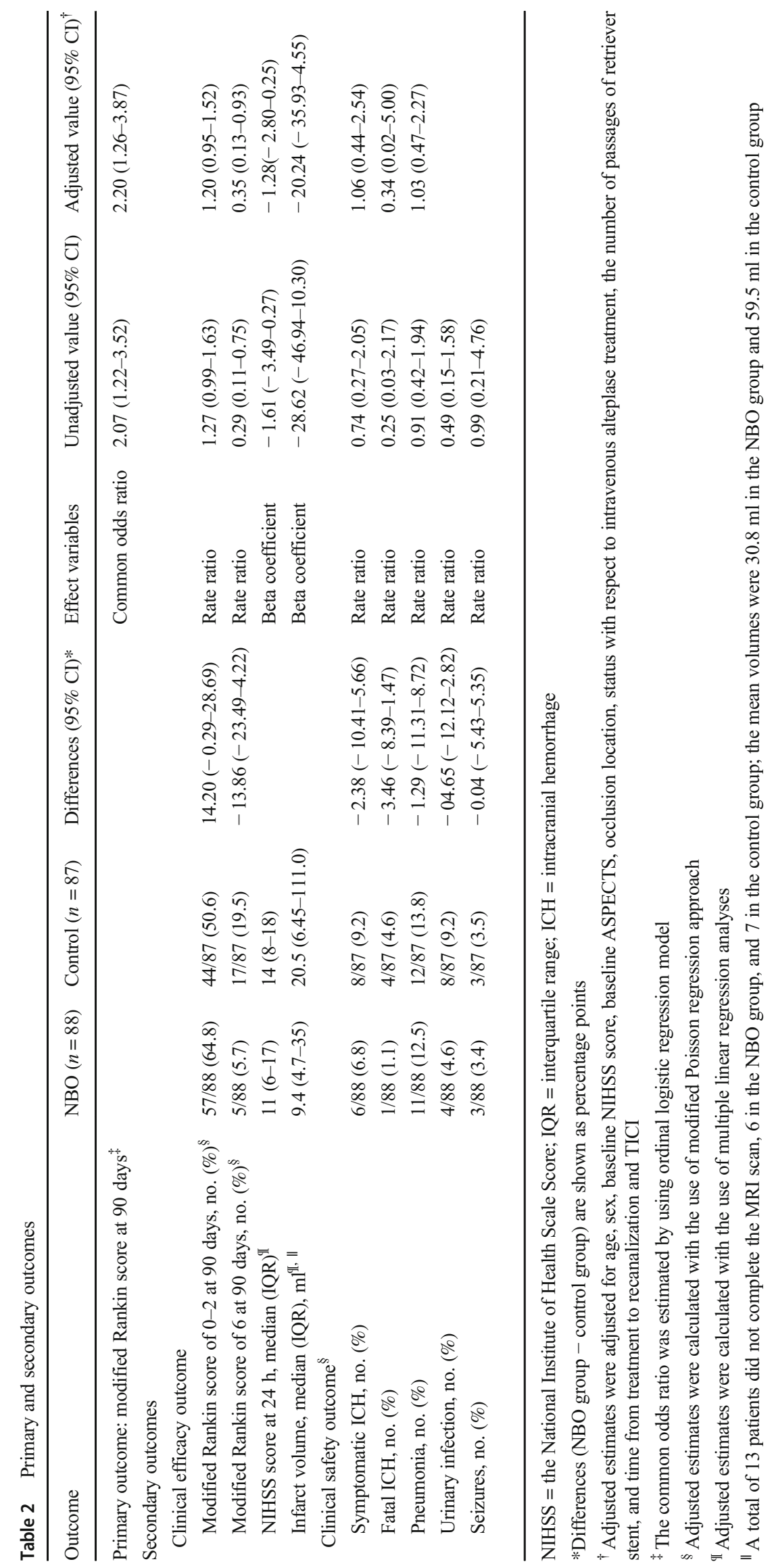




\section{Infarct Volume}

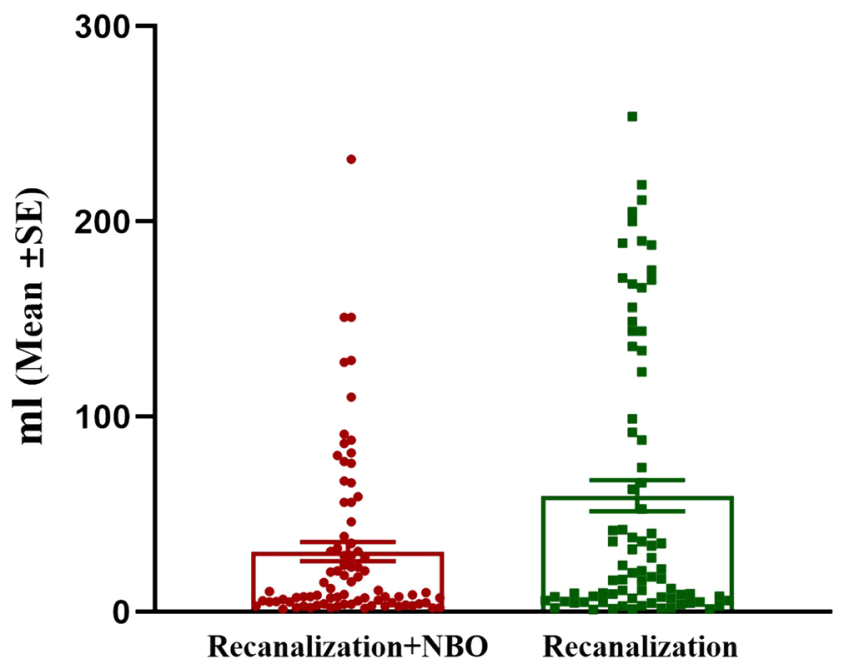

Fig. 3 Infarct volume at $24 \mathrm{~h}$ after vessel recanalization. Comparison on infarct volume at $24 \mathrm{~h}$ after vessel recanalization between the 2 groups, demonstrating a significant reduction of infarct volume determined by MRI in the NBO group

reported NBO as a harmful adjuvant in AIS without endovascular thrombectomy. Thus, its routine use in nonhypoxic stroke victims with minor or moderate stroke burden has been discouraged [27]. A recent prospective observational cohort of ICU (intensive care unit) admitted AIS LVO patients following endovascular therapy evaluated treatment with hyperoxia $\left(\mathrm{PaO}_{2}\right.$ $>120 \mathrm{mmHg}$ via endotracheal tube or face mask $\mathrm{FiO}_{2}$ 50\%) [28]. The authors noted a higher mortality rate and worse functional outcomes in the hyperoxia group as compared to the control group $\left(\mathrm{PaO}_{2}<120 \mathrm{mmHg}\right)$. In this study, all patients were intubated for mechanical thrombectomy and the use of hyperoxia was determined by a single test of blood gases prior to ICU admission. However, $66 \%$ of patients in the hyperoxia group remained intubated at ICU admission compared to only $45 \%$ of those in the control group. Thus, induction of general anesthesia, the method of oxygen supplementation (endotracheal tube), and the medications for ventilation comfort may have contributed to the authors' negative findings. In contrast, oxygen administration by simple Venturi mask with high-flow oxygen in our study was found to be safe and efficacious after endovascular recanalization.

Efficacy of NBO and the Key Role of Reperfusion Oxygen can easily diffuse across the blood-brain barrier (BBB) and thus carries the potential to improve outcomes by ameliorating the side effects associated with hypoxic episodes and resultant secondary brain injury [15]. Previous studies have failed to establish a clear significant neuroprotective effect of NBO administration in stroke patients. However, in the study, the efficacy of NBO in AIS patients after vessel recanalization was suggested by significantly improving functional outcome and decreasing mortality with a smaller infarct volume compared to AIS patients treated with low-flow oxygen, consistent with the preliminary data on outcome [29]. It should be noted that there was no requirement for endovascular recanalization in previous clinical studies [21, 23, 30-32]. Additionally, a recently published paper in JAMA [33] suggested that lowflow NBO did not reduce disability or mortality at 90 days with uncertain recanalization status in the subgroup analysis on the ischemic stroke patients. Unlike previous studies, NBO as an adjuvant therapy was implemented after endovascular thrombectomy in this study. As such, the process of revascularization and resultant reperfusion may be helpful and necessary to augment the neuroprotective effect of NBO.

Importance of High-Flow in NBO Therapy Previous studies, in which low-flow NBO has been ineffective, have hypothesized that high-flow NBO may be more effective $[21,30]$. Early administration of high-flow oxygen may help maintain the viability of the ischemic penumbra. Mask devices are the most common modalities used to supplement high-flow NBO in clinical trials [21, 34]. Venturi masks can supply higher flow and concentrations of oxygen when compared to nasal devices. A metaanalysis involving 11 randomized controlled trials revealed that patients benefited from mask-administered NBO compared to those treated by nasal cannula-NBO, suggesting that mask administration was more feasible and beneficial among stroke patients [21]. Chiu et al. demonstrated that patients benefited most from Venturi mask-NBO following severe AIS [30]. Therefore, administration of high-flow NBO by Venturi mask should be considered to augment the therapeutic efficacy of revascularization in LVO patients as was observed in the present study.

NBO Supplementation with an $\mathrm{FiO}_{2} 50 \%$ for a Duration of $6 \mathrm{~h}$ The optimal duration for administration of NBO in AIS remains to be determined. Although NBO was administrated from ischemia to reperfusion, a recent rat study demonstrated that an 8-h NBO treatment exhibited an optimal neuroprotective effect as compared to a 2- or 4-h duration of treatment [35]. However, long term NBO $(60 \mathrm{~h})$ was found to be harmful in the brains of guinea pigs [36]. Previous clinical trials have utilized a range of NBO supplementation of 8 to $24 \mathrm{~h}$ duration and $27-60 \% \mathrm{FiO}_{2}$ in AIS patients, albeit without vessel recanalization; $100 \% \mathrm{FiO}_{2} \mathrm{NBO}$ has been reported rarely in clinical trial [21]. In one clinical registry study (ClinicalTrials.gov Identifier: NCT00414726) on NBO therapy in AIS, $30-45 \mathrm{ml} / \mathrm{min}$ flow of oxygen supplementation via facemask was used as compared to $15 \mathrm{ml} / \mathrm{min}$ in our study [32]. Although the blinded external medical monitor did not adjudicate any deaths as "definitely" 


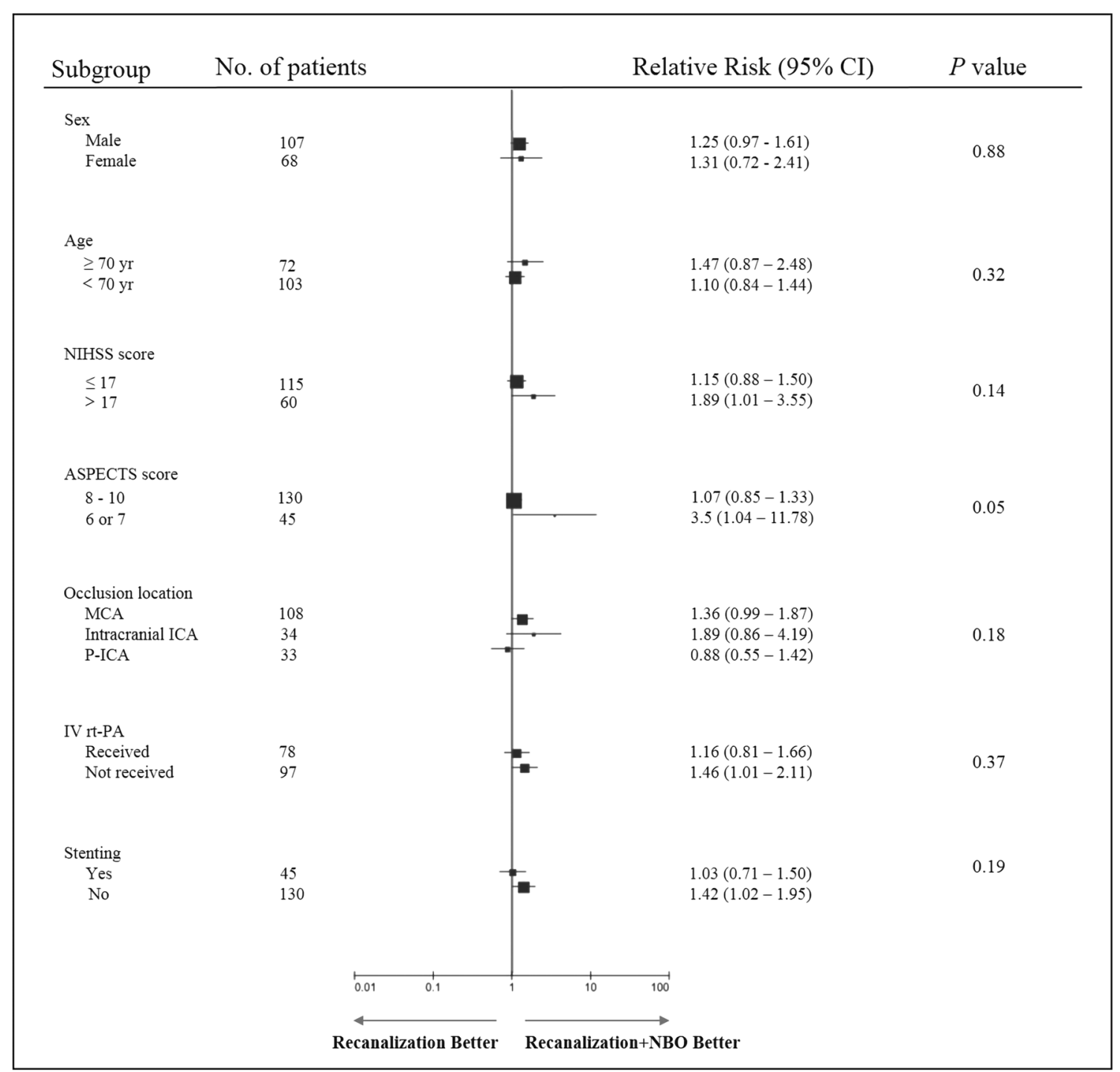

Fig. 4 Analysis of functional independence at 90 days in subgroups. Functional independence was defined as a score on the modified Rankin Scale of 0,1 , or 2. $p$ values were based on the Breslow-Day test for homogeneous odds ratios across subgroups. Squares indicate point estimates for treatment effects, and the size of the square is proportional to the precision of the estimate. NIHSS $=$ scores on the National Institutes of Health Stroke Scale, range from 0 to 25, with higher scores indicating

or "probably" related to therapy, the study was unsuccessfully terminated in 2009. The safety of excessive oxygen supplement in AIS patients remained unclear. Because the present study was the first to administer NBO in AIS patients after vessel recanalization with mechanical thrombectomy, moderate administration with a duration of $6 \mathrm{~h}$ and $50 \% \mathrm{FiO}_{2} \mathrm{NBO}$ by Venturi mask was chosen as the primary oxygenation strategy for safety and feasibility purpose. Although this provided positive results, the optimal duration and oxygen concentration remains to be determined to maximize outcomes in future studies. It is important to note that the timing, duration, and volume of NBO administration in conjunction with the degree more severe neurologic deficits, the threshold of 17 was the threshold used in stratifying randomization; ASPECTS = Alberta Stroke Program Early CT Score, ranges from 6 to 10, with higher scores indicating a smaller infarct core; $\mathrm{MCA}=$ middle cerebral artery segment; ICA = internal carotid artery; P-ICA = proximal segment of internal carotid artery occlusion combined with intracranial large artery occlusion; IV rt-PA = intravenous recombinant tissue plasminogen activator

of vessel recanalization, together play a role in neuroprotection for stroke caused by LVO.

\section{Limitations and Future Studies}

Our study has several limitations. First, this trial was performed in a single center. Second, high-flow NBO was administrated only after vessel recanalization in this study and future studies may consider initiation of the high-flow NBO supplementation prior to reperfusion therapy. Third, absence of adjusted infarct volumes based on baseline data may 
weaken our findings on neuroprotection of NBO to some degree. To comprehensively understand the beneficial effect of NBO, baseline DWI with perfusion and collateral data, allowing for adjustment and more accurate assessment of infarct volumes will be considered in future studies (on this note, the initial ASPECTS scores were similar between groups, as was the time from symptom onset to recanalization, thus likely our DWI data is accurate to some degree). Fourth, due to the concern of arterial blood gases may complicate this simple therapy, oxygen concentration in the blood was not monitored before or during the NBO process. However, it cannot be ruled out that more patients possibly presenting with hypoxia were in the NBO arm. The data on the pulmonary post-EVT/ tPA course should have been analyzed in details, in order to further determine the mechanisms underlying beneficial effect of NBO. The present study strongly suggested a therapeutic potential of NBO for AIS after endovascular recanalization. It thus warrants a multicenter study with large sample to demonstrate the generalization of this finding. It is very likely that we will further prove the beneficial effect of NBO in ischemic stroke with recanalization, especially when the limitations are considered.

\section{Conclusion}

The result suggests that high-flow NBO therapy after endovascular recanalization is safe and may be effective in improving functional outcomes and reducing mortality and infarct volumes in anterior circulation LVO stroke patients within $6 \mathrm{~h}$ from stroke onset. Vessel recanalization before initiation of NBO may augment the efficacy of NBO in providing neuroprotection following AIS.

Supplementary Information The online version contains supplementary material available at https://doi.org/10.1007/s13311-020-00979-3.

Acknowledgments: We would like to show my deepest gratitude to Hongqiu Gu, PhD, from China National Clinical Research Center for Neurological Diseases and $\mathrm{Yu}$ Cao, PhD, from the Department of Science and Education in Beijing Luhe Hospital Capital Medical University who were involved in the analysis plan and execution.

Required Author Forms Disclosure forms provided by the authors are available with the online version of this article.

Funding The present study was partially supported by the National Nature and Science Foundation of China (81871838) to Xiaokun Geng and Beijing Municipal Science Technology Commission (Z151100003915134) to Huishan Du.

\section{Compliance with ethical standards}

Disclosures The authors report no disclosures relevant to the manuscript.

\section{References}

1. Goyal M, Menon BK, van Zwam WH, Dippel DW, Mitchell PJ, Demchuk AM, et al. Endovascular thrombectomy after large-vessel ischaemic stroke: a meta-analysis of individual patient data from five randomised trials. Lancet. 2016;387(10029):1723-31.

2. Malhotra K, Liebeskind DS. Wake-up stroke: dawn of a new era. Brain Circ 2016;2(2):72-9.

3. Jadhav AP, Jovin TG. Endovascular therapy for acute ischemic stroke: the standard of care. Brain Circ 2016;2(4):178-82.

4. Rajah GB, Ding Y. Experimental neuroprotection in ischemic stroke: a concise review. Neurosurg Focus 2017;42(4):E2.

5. Ginsberg MD. Neuroprotection for ischemic stroke: past, present and future. Neuropharmacology. 2008;55(3):363-89.

6. Powers WJ, Rabinstein AA, Ackerson T, Adeoye OM, Bambakidis $\mathrm{NC}$, Becker K, et al. Guidelines for the early management of patients with acute ischemic stroke: 2019. update to the 2018 guidelines for the early management of acute ischemic stroke: a guideline for healthcare professionals from the American Heart Association/ American Stroke Association. Stroke. 2019: STR0000000000000211.

7. Babadjouni RM, Walcott BP, Liu Q, Tenser MS, Amar AP, Mack WJ. Neuroprotective delivery platforms as an adjunct to mechanical thrombectomy. Neurosurg Focus 2017;42(4):E4.

8. Savitz SI, Baron JC, Yenari MA, Sanossian N, Fisher M. Reconsidering neuroprotection in the reperfusion era. Stroke. 2017;48(12):3413-9.

9. Hill MD, Goyal M, Menon BK, Nogueira RG, McTaggart RA, Demchuk AM, et al. Efficacy and safety of nerinetide for the treatment of acute ischaemic stroke (ESCAPE-NA1): a multicentre, double-blind, randomised controlled trial. Lancet. 2020;395(10227):878-87.

10. Geng Xk, Elmadhoun O, Peng CY, Ji XM, Hafeez A, Liu Z, et al. Ethanol and normobaric oxygen: novel approach in modulating pyruvate dehydrogenase complex after severe transient and permanent ischemic stroke. Stroke. 2015;46(2):492-9.

11. Thibodeau A, Geng XK, Previch LE, Ding YC. Pyruvate dehydrogenase complex in cerebral ischemia-reperfusion injury. Brain Circ 2016;2(2):61-6.

12. Beker MC, Caglayan AB, Kelestemur T, Caglayan B, Yalcin E, Yulug B, et al. Effects of normobaric oxygen and melatonin on reperfusion injury: role of cerebral microcirculation. Oncotarget. 2015;6(31):30604-14.

13. Esposito E, Mandeville ET, Hayakawa K, Singhal AB, Lo EH. Effects of normobaric oxygen on the progression of focal cerebral ischemia in rats. Exp Neurol 2013;249:33-8.

14. Yang D, Ma L, Wang P, Yang D, Zhang Y, Zhao X, et al. Normobaric oxygen inhibits AQP4 and NHE1 expression in experimental focal ischemic stroke. Int J Mol Med 2019;43(3):1193-202.

15. Singhal AB. A review of oxygen therapy in ischemic stroke. Neurol Res 2007;29(2):173-83

16. Bigdeli MR, Rasoulian B, Meratan AA. In vivo normobaric hyperoxia preconditioning induces different degrees of antioxidant enzymes activities in rat brain tissue. Eur J Pharmacol 2009;611(13):22-9.

17. Ejaz S, Emmrich JV, Sitnikov SL, Hong YT, Sawiak SJ, Fryer TD, et al. Normobaric hyperoxia markedly reduces brain damage and sensorimotor deficits following brief focal ischaemia. Brain. 2016;139(Pt 3):751-64

18. Flynn EP, Auer RN. Eubaric hyperoxemia and experimental cerebral infarction. Ann Neurol 2002;52(5):566-72.

19. Geng XK, Fu P, Ji XM, Peng CY, Fredrickson V, Sy C, et al. Synergetic neuroprotection of normobaric oxygenation and ethanol in ischemic stroke through improved oxidative mechanism. Stroke. 2013;44(5):1418-25. 
20. Cai LP, Stevenson J, Geng XK, Peng CY, Ji XM, Xin R, et al. Combining normobaric oxygen with ethanol or hypothermia prevents brain damage from thromboembolic stroke via PKC-AktNOX modulation. Mol Neurobiol 2017;54(2):1263-77.

21. Ding J, Zhou D, Sui M, Meng R, Chandra A, Han J, et al. The effect of normobaric oxygen in patients with acute stroke: a systematic review and meta-analysis. Neurol Res 2018;40(6):433-44.

22. Padma MV, Bhasin A, Bhatia R, Garg A, Singh MB, Tripathi M, et al. Normobaric oxygen therapy in acute ischemic stroke: a pilot study in Indian patients. Ann Indian Acad Neurol 2010;13(4):2848 .

23. Singhal AB, Benner T, Roccatagliata L, Koroshetz WJ, Schaefer PW, Lo EH, et al. A pilot study of normobaric oxygen therapy in acute ischemic stroke. Stroke. 2005;36(4):797-802.

24. Hong KS, Saver JL. Quantifying the value of stroke disability outcomes: WHO global burden of disease project disability weights for each level of the modified Rankin Scale. Stroke. 2009;40(12):382833.

25. Tung EL, McTaggart RA, Baird GL, Yaghi S, Hemendinger M, Dibiasio EL, et al. Rethinking thrombolysis in cerebral infarction $2 \mathrm{~b}$ : which thrombolysis in cerebral infarction scales best define near complete recanalization in the modern thrombectomy era? Stroke. 2017;48(9):2488-93.

26. Zou G. A modified Poisson regression approach to prospective studies with binary data. Am J Epidemiol 2004;159(7):702-6.

27. Ronning OM, Guldvog B. Should stroke victims routinely receive supplemental oxygen? A quasi-randomized controlled trial. Stroke. 1999;30(10):2033-7.

28. Lopez HV, Vivas MF, Ruiz RN, Martinez JR, Navaridas BG, Villa $\mathrm{MG}$, et al. Association between post-procedural hyperoxia and poor functional outcome after mechanical thrombectomy for ischemic stroke: an observational study. Ann Intensive Care 2019;9(1):59.
29. Geng XK, Cheng Z, Dornbos D, Du HS, Ding YC. High flow normobaric oxygen (NBO) therapy provides effective neuroprotection after endovascular recanalization in acute ischemic stroke: Stroke; 2019. Available from: https://doi.org/10.1161/str.50. suppl_1.tmp8. Accessed 30 Jan 2019

30. Chiu EH, Liu CS, Tan TY, Chang KC. Venturi mask adjuvant oxygen therapy in severe acute ischemic stroke. Arch Neurol 2006;63(5):741-4.

31. Mazdeh M, Taher A, Torabian S, Seifirad S. Effects of normobaric hyperoxia in severe acute stroke: a randomized controlled clinical trial study. Acta Med Iran 2015;53(11):676-80.

32. Poli S, Baron JC, Singhal AB, Hartig F. Normobaric hyperoxygenation: a potential neuroprotective therapy for acute ischemic stroke? Expert Rev Neurother 2017;17(12):1131-4.

33. Roffe C, Nevatte T, Sim J, Bishop J, Ives N, Ferdinand P, et al. Effect of routine low-dose oxygen supplementation on death and disability in adults with acute stroke: the Stroke Oxygen Study Randomized Clinical Trial. JAMA. 2017;318(12):1125-35.

34. Shi SH, Qi ZF, Luo YM, Ji XM, Liu KJ. Normobaric oxygen treatment in acute ischemic stroke: a clinical perspective. Med Gas Res 2016;6(3):147-53.

35. Yuan Z, Pan R, Liu W, Liu KJ. Extended normobaric hyperoxia therapy yields greater neuroprotection for focal transient ischemiareperfusion in rats. Med Gas Res 2014;4:14.

36. Tatarkova Z, Engler I, Calkovska A, Mokra D, Drgova A, Hodas P, et al. Effect of long-term normobaric hyperoxia on oxidative stress in mitochondria of the guinea pig brain. Neurochem Res 2011;36(8):1475-81.

Publisher's Note Springer Nature remains neutral with regard to jurisdictional claims in published maps and institutional affiliations. 